\title{
The Obsessive Compulsive Disorder Symptoms after the Successful Treatment of the Pineal Germinoma: A Case Report
}

\author{
Aslihan Okan Ibiloglu ${ }^{1}$, Abdullah Atli ${ }^{2}$, Esref Akil ${ }^{3}$, Suleyman Demir ${ }^{1}$ and Mahmut Bulut ${ }^{2}$ \\ 1. Assistant Professor Doctor, Department of Psychiatry, Dicle University, Medicine Faculty, Diyarbakir 21280 Turkey \\ 2. Associate Professor, Department of Psychiatry, Dicle University, Medicine Faculty, Diyarbakir 21280 Turkey \\ 3. Associate Professor, Department of Neurology, Dicle University, Medicine Faculty, Diyarbakir 21280 Turkey
}

\begin{abstract}
We report here, a young male patients referred with "Obsessive Compulsive Disorder" symptoms which emerged after the successful treatment of pineal germinoma. OCD (obsessive-compulsive disorder) is a frequent, chronic, and clinically disorder which may presents in several neurologic disorders, especially occurs, in early adult life. Essential features of OCD are obsessional thoughts, compulsive acts as the ritualistic behavior, anxiety, and specific cognitive impairments. The cause of obsessive-compulsive disorder isn't fully understood. One of the many theories of the pathophysiology about to OCD is includes with hyperactivity in certain subcortical and cortical regions of brain also, dysfunction of the cortico-striatal circuits, particularly implicated in orbitofrontal cortices and basal ganglions. Additionally, pineal gland functioning is remarkable for the mental health disorders, particularly in OCD. On the basis of the investigations to present case report, we discussed the probable reasons of OCD symptoms, emphasizing the role of pathophysiology including the cortico subcortical pathways in genesis of the symptoms.
\end{abstract}

Key words: Pineal gland, germinoma, obsessive compulsive disorder, early adult life.

\section{Introduction}

Pineal gland is located in epithalamus which between to two hemispheres, situated through a groove where the two halves of the join in thalamus, near the center of the brain, [1]. Recently, pineal gland functioning is remarkable particularly in OCD (obsessive-compulsive disorder) $[1,2]$. Intracranial germinoma is a type of germ cell tumor which most common location in the pineal or supra sellar regions. Also, intracranial germinomas are most commonly diagnosed between ages of 12 to 20 , predominantly affected young males than females [2,3].

OCD is a neuropsychiatric disorder characterized by the presence of recurrent, intrusive and distressing thoughts, or images, and urges to action. Recent evidence suggest that OCD patients are exhibited

Corresponding author: Aslihan Okan Ibiloglu, M.D., assistant professor doctor, Dicle University, Medicine Faculty, Department of Psychiatry 21280, Diyarbakir, Turkey. various pathological behaviors within associative dysfunction of the frontal lobe and/or basal gangliona $[1,3]$. The etiology and pathophysiology of OCD are not fully understood. Various factors involving psychosocial, genetic, neuroanatomical pathways (cortico-striatal-thalamic-cortical pathways), and neurochemical factors have been identified [1-3]. We presented here, a rare case referred with OCD symptoms resulting after the successful treatment of a pineal germinoma.

\section{Case Presentation}

We present here, a case of 19 years old, poor and single Turkish young man, who lived with his parents and daughters, in Diyarbakir, Turkey. He presented to us with 2-year history of obsessive symptoms including the sexuality and contamination, additionally compulsive complaints. Especially, his symptoms are centred on washing and controlling. 
Approximately 2 years before presenting to our clinic, he was diagnosed with a pineal germinoma. According to the medical records, pineal germinoma was completely resected also, surgical specimens and histopathologic examination confirmed with pineal germinoma. He was in remission, following after completion of the germinoma resection treatment with chemotherapy. He denied experiencing any OCD symptoms or other psychiatric symptoms, prior to this diagnosis. Moreover, he showed a striking onset of OCD symptoms. The patient's symptoms become gradually worse coexisting presence of sexual with contamination thinking. The history of family together with his psychiatric and neurologic history was unremarkable. When he was presented to our clinic, he did not use any drugs.

Detailed physical and neurological examinations revealed, including MR images of the brain, a stable appearance without evidence of tumor recurrence, and no other structural changes in his brain. Serum chemistry and complete blood count were normal.

At the psychiatric examination, his mood is severely anxious and low, including the anhedonia and sadness. He has unable to stay alone, on the other hand his obsessive and compulsive complaints are accompanied by his repeated intrusive thoughts, about to cleanliness and washing.

The Y-BOCS (Yale-Brown Obsessive Compulsive Scale) $[4,5]$ was completed during at the pretreatment and posttreatment. On the Y-BOCS, he scored 36 on a 0-40 scale, indicating extremely severe of symptoms. After treating sertraline $300 \mathrm{mg} /$ day with risperidone 3 $\mathrm{mg}$ /day, his obsessive and compulsive symptoms were completely improved, throughout the one year. Hence, there was a dramatic reduction according to baseline to follow-up in Y-BOCS total scores decreasing by over 25 points.

\section{Discussion}

OCD is a frequent, chronic, and disabling disorder which characterized the obsessions (intrusive, repetetive, and persistent thoughts or images), compulsions (repetetive and ritualistic behaviors or mental acts), anxiety, and specific cognitive impairments on the other hand it may presents in several neurologic disorders, especially occurs in early adult life [1].

In literature, many neurologic disorders are accompanied by the OCD symptoms, as in present case $[2,3]$.Many disorders may result in the emergence of the substantial OCD symptoms; that can be listed as traumatic brain injury, cerebral infarction, brain tumors, epilepsy, encephalitis, developmental disorders, diabetes insipidus, multiple sclerosis and acute intermittent porphyria [1]. In literature, melatonin disturbance has been reported in OCD [6]. As noted, melatonin which produced in pineal gland as a serotonine derived hormone may alter circadian rhythm with serotonin metabolism [7].

There are many case reports, which confirming to our case, documenting the relation of OCD symptoms with progression of intracranial tumor or remission, after the successful treatment $[2,3,6]$. Also, as in many cases, OCD symptoms started after successful surgical removal or successful radiation treatment of brain tumors. Recently, dysfunction of cortico-striatal circuits, particularly orbito-frontal cortices and basal ganglionas has been implicated in pathophysiology of OCD $[1,2]$. These features provide a circuit mechanism for adjusting emotional drive in processes disrupted in various psychiatric disorders, such as phobias and OCD $[2,3]$.

Maybe, the timing of symptom onset suggests a potential relationship of OCD symptoms with pineal body functioning which affected from intracranial germinoma resection [2, 3]. Maybe, an alternative explanation for developing OCD symptoms in our patient may have delayed effect which resulting from either intracranial tumor or from the surgical treatment in the regions, such as basal ganglia, anterior cingulate, and orbito-frontal cortex [2, 3, 8]. Furthermore, our patient had no experience of psychiatric symptoms 

of the Pineal Germinoma: A Case Report

before the onset of brain tumor. As noted, these findings further implicate pineal body dysfunction in the pathophysiology of OCD $[2,6]$.

\section{Conclusions}

In our opinion, this case showed an interesting relationship between OCD and intracranial tumors, so that complaints of OCD appeared when the pineal germinoma was detected.

\section{Consent}

Written informed consent was obtained from the patient for publication of this case report.

\section{References}

[1] Zohar, Joseph, ed. 2012. "Obsessive Compulsive Disorder: Current Science and Clinical Practice." Hoboken, N. J., USA: John Wiley and Sons, 2012. ProQuest ebrary.

[2] De Nadai, A. S., Storch, E. A., and Alvaro, J. L. 2011. "Development of Obsessive-Compulsive Disorder Following a Pineal Germinoma: A Case Report.” Am. J. Psychiatry 168 (5): 550-1.
[3] Mordecai, D., Shaw, R. J., Fisher, P. G., et al. 2000. "Case Study: Suprasellar Germinoma Presenting with Psychotic and Obssessive-Compulsive Symptoms." J. Am. Acad. Child Adolesc Psychiatry 39 (1): 116-9.

[4] Goodman, W. L., Price, L. H., Rasmussen, S. A., and Mazure, C. 1989. "The Yale-Brown Obsessive Compulsive Scale (Y-BOCS): Validity.” Arch. Gen. Psychiatry 4: 1012-6.

[5] Karamustafalığlu, O. K., Üçışık, A. M., and Ulusoy, M. ve ark. 1993. "Yale-Brown Obsesyon-Kompülsiyon Derecelendirme Ölçeğinin Geçerlik ve Güvenilirlik Çalışması. 29." Ulusal Psikiyatri Kongresi Program ve Bildiri Özetleri Kitabı, Bursa. Savaş Ofset, s: 86.

[6] Monteleone, P., Catapano, F., Del Buono, G., and Maj, M. 1994. "Circadian Rhythms of Melatonin, Cortisol, and Prolactin in Patients with Obsessive Compulsive Disorder." Acta. Psychiatr. Scand. 89: 411-5.

[7] Miguez, J. M., Martin, F. J., and Aldegunde, M. 1987. "Melatonin Effects on Serotonin Synthesis and Metabolism in the Striatum, Nucleus Accumbens, and Dorsal and Median Raphe Nuclei of Rats." Neurochem Res. 22: 87-92.

[8] Gamazo-Garrán, P., Soutullo, C. A., and Ortuño, F. 2002. "Obsessive-Compulsive Disorder Secondary to Brain Dysgerminoma in an Adolescent Boy: A Positron Emission Tomography Case Report." J. Child Adolesc Psychopharmacol. 12 (3): 259-63. 